\title{
Application of Quadratic Rotation-Orthogonal Composite Experimental Design to Assess the Relationship between Growth Environment and Ultraweak Luminescence of Mint
}

\author{
Yao Zhan ${ }^{1}$, Yi Lin ${ }^{1}$, Chunfang Wang ${ }^{1}$, Jianping $\mathrm{Li}^{1}$, and Yong $\mathrm{Yu}^{2, *}$ \\ ${ }^{1}$ College of Biosystems Engineering and Food Science, Zhejiang University, \\ Hangzhou, Zhejiang, 310058 \\ ${ }^{2}$ College of Biosystems Engineering and Food Science, Zhejiang University, \\ Hangzhou, Zhejiang, 310058, China \\ yyuzju@zju.edu.cn
}

\begin{abstract}
Quadratic rotation-orthogonal composite experimental design was applied in this experiment to obtain the ultraweak luminescence property of mint affected by multilateral growth environmental factors including illumination, temperature, humidity, and moisture. The influence models of four factors on luminescence were established. Analysis showed that different factors and their interactions had different effect on luminous value, among which the interaction of illumination and humidity had the most significant influence on luminescence of mint $(\mathrm{p} \leq 0.01)$. And the maximum luminous value was obtained when ten stick of lamp tube (intensity of illumination value $4229 \mathrm{Lx}$ ) was used, mean temperature, humidity and water were $35^{\circ} \mathrm{C}, 85 \%$ and $90 \mathrm{~mL} / \mathrm{d}$ respectively. On the base of that, linear effects and their interactions that affected ultraweak luminescence were also studied.
\end{abstract}

Keywords: mint, growth environment, ultraweak luminescence.

\section{Introduction}

It is one century since people know the ultraweak luminescence of plants. Although the mechanism has not been completely revealed, researches have already demonstrated that ultraweak luminescence is a dynamic indicator that reflects the metabolism, function mediation and information exchange inner and outer the cells in organisms [1-2]. The discovery of the intimate positive correlation between plant luminescence and vitality is one of the most basic achievements and is one of the most important detecting techniques applied in modern farming management [3].

For instance, viability and luminescence of soybeans were improved significantly after electromagnetic radiation. But the viability of Mung bean was brought down by

\footnotetext{
* Corresponding author. 
using metabolic inhibitor, and the same as the luminescence [4]. Similar consequences were obtained in previous studies with rice, peanut leaf and spinach [5].

On the basis of that, many researches aiming at viability support and detection were done to study rules and factors that influence the bio-photon emission. Plant's viability and luminescence are affected by some physical (oxygen, temperature, irradiation, etc.) [6-9], chemical (oxidant, metabolic inhibitor, etc.) factors [10], hormone and environmental stress [11-13]. Benefited from the development of electronic and information technology, the alteration of plant's luminescence will be identified preferentially before the change of viability property is observed. It facilitates the application of ultraweak luminescence technology in the fields of industrial seeding nursery, intelligent cultivation and breed.

However, the study of plant's ultraweak luminescence is not complete, especially the luminescence properties affected by multilateral growth environment factors, and the effect of interaction of different factors is not clear to plant luminescence, which significantly restricts the application in the fields mentioned before.

In this article, quadratic rotation-orthogonal composite experimental design was used to study the luminescence properties of mint that affected by multilateral growth environment factors, and the relationship between luminescence properties and different factors and their interaction was explored. Correlative knowledge was prepared to facilitate the application of ultraweak luminescence testing technology in modern farming management.

\section{Materials and Methods}

\subsection{Materials and Processing}

15 bonsai of mints were supplied by Zhongtai nursery stock base of Hangzhou New Blue-Sky Landscape Group, mints grew in a greenhouse with identical environment and nutrient conditions for 45 days, and the degrees of dense foliage were kept similar. Mints were put into an artificial climate incubator (Artificial Climate Box, RXZ) after collected, kept in $30^{\circ} \mathrm{C}$ and $90 \%$ in humidity and exposed in light before use. The experiment was carried out on three replicates.

\subsection{Range of Environment Factors}

The environment factors of osmanthus tree seedings include illumination, temperature, relative humidity and moisture. These factors were controlled by RXZ except for moisture that artificially controlled by using a injector with scales. Ranges of these four factors were formulated as follows:

Illumination: according to the average illumination of the greenhouse, illuminance was regulated by ten $40 \mathrm{~W}$ fluorescent tubes, intensity was controlled by opening different numbers of tubes (intensity of one $40 \mathrm{~W}$ fluorescent tube is $422.9 \pm 10.1 \mathrm{Lx}$ ).

Temperature: the temperature range was automatically controlled by $\mathrm{RXZ}$ at $15^{\circ} \mathrm{C} \sim$ $35^{\circ} \mathrm{C}$, accuracy was $\pm 0.5^{\circ} \mathrm{C}$. 
Humidity: the relative humidity was controlled by RXZ at $65 \% \sim 85 \%$, accuracy is $\pm 1 \%$.

Moisture: according to the moisture range $(10 \sim 90 \mathrm{~mL} / \mathrm{d})$ obtained from preliminary test, moisture was controlled artificially by using a injector with scales at the same time of everyday.

\subsection{Measurement of Ultraweak Luminescence Value}

The optimum detect condition of ultraweak luminescence was obtained from pre-experiment. mint was cultivated in given conditions for 3 days before test, $0.2 \mathrm{~g}$ of mint (leaves) was detected under $35^{\circ} \mathrm{C}$ to get the maximum luminescence value.

Photo-induced delayed luminescence was observed at the beginning of test, emission of mint leaves in constant time interval decreased rapidly and waved evidently. After $3 \sim 5 \mathrm{~min}$, delayed luminescence disappeared gradually, emission tended to be steady. Hence, the whole data acquisition time of each experiment was 10 min, and data of last 5 min was adopted (detection cup without samples as control).

\subsection{Experimental Design and Data Analysis}

Quadratic rotation-orthogonal composite experimental design was used to analyze the effect of illumination, temperature, relative humidity, moisture, and interaction to the luminescence of samples. Quadratic rotation-orthogonal composite experimental design in coded and actual level of variables and the luminous value of each sample were detected (data not shown). 36 experiments were carried out with 12 replicates at the center point.

The result was analyzed to build the model of environmental factors to ultraweak luminescence by using Data Processing System (DPS), the optimum values of environment variables were obtained when luminous value was maximum, and the effects of influential factors and interactions on luminescence of mint were further studied.

\section{Results and Analysis}

\subsection{Regression Analysis and Optimal Parameter Analysis}

Polynomial regression equation with linear, quadratic and interaction terms was obtained by using DPS, analysis of variance (ANOVA) of each effect was shown in Tab.1.

Tab. 1 showed effects of single factors and interactions of four environment factors of four environment variables on the luminous value of mint. the regression equation of response function had a high correlation coefficient $(\mathrm{r}=0.993)$, can be used to predict luminous value of mint in trial stretch. 
Table 1. Model and condensed ANOVA (analysis of variance) table in coded level of variables

\begin{tabular}{ccc}
\hline $\begin{array}{c}\text { Source of } \\
\text { variations }\end{array}$ & $\begin{array}{c}\text { Coefficient of } \\
\text { polynomial }\end{array}$ & F-Value \\
\hline$x_{1}$ & 1.83119 & \\
$x_{2}$ & 0.04471 & $0.03496^{\mathrm{NS}}$ \\
$x_{3}$ & 0.12061 & $0.25445^{\mathrm{NS}}$ \\
$x_{4}$ & -0.62317 & $6.79296^{* *}$ \\
$x_{1}^{2}$ & 0.67242 & $7.90896^{* *}$ \\
$x_{2}^{2}$ & 0.48344 & $5.45088^{* *}$ \\
$x_{3}^{2}$ & 0.07280 & $0.12361^{\mathrm{NS}}$ \\
$x_{4}^{2}$ & 0.44216 & $4.55968^{* *}$ \\
$x_{1} x_{2}$ & 0.48463 & $5.47771^{* *}$ \\
$x_{1} x_{3}$ & 0.14325 & $0.23931^{\mathrm{NS}}$ \\
$x_{1} x_{4}$ & -0.84385 & $8.30386^{* * *}$ \\
$x_{2} x_{3}$ & 0.54536 & $3.46835^{*}$ \\
$x_{2} x_{4}$ & 0.20839 & $0.50640^{\mathrm{NS}}$ \\
$x_{3} x_{4}$ & 0.77622 & $7.02619^{* *}$ \\
$r$ & -0.08052 & $0.07561^{\mathrm{NS}}$ \\
\hline$x_{3}$ & 0.933 & - \\
\hline
\end{tabular}

Note: Variables: $x_{1}$-light, $x_{2}$-temperature, $x_{3}$-relative humidity, $x_{4}$-moisture.

NS Non-significant at $p>0.10$.

* Significant at $p \leq 0.10$.

** Significant at $p \leq 0.05$.

*** Significant at $p \leq 0.01$.

To determine the value of four factors including illumination, temperature, moisture and humidity required by mint, optimal calculation of luminous value was carried out based on the intimate positive correlation between luminous value and viability of plants. Results showed that the maximum luminous value of mint (18.7 photon/s) was acquired when 10 lamps were used (illumination value was $4229 \mathrm{Lx}$ ), temperature, humidity and moisture were $35{ }^{\circ} \mathrm{C}, 65 \%$ and $90 \mathrm{~mL} / \mathrm{d}$ respectively. Because of the intimate positive correlation between luminous value and viability of plants, viability of mint was higher under the optimum environmental condition than that under any other conditions. 


\subsection{Analysis of Effect of Each Factor and Interaction on Ultraweak Luminous Value}

In order to facilitate further analysis of single factors and interaction effects on luminous value, the regression model shown in Tab.1 was corrected. First, non-significant terms in Tab.1 were rejected, the rest of terms (coded level) constitute the relational model with the luminous value of mint (Eq.1). The correlation coefficient was further elevated $(\mathrm{r}=0.953)$.

$$
\begin{gathered}
Y=1.83119-0.62317 x_{3}+0.67242 x_{4}+0.48344 x_{1}^{2}+0.44216 x_{3}^{2}+0.48463 x_{4}^{2}-0.84385 x_{1} x_{3} \\
+0.54536 x_{1} x_{4}+0.77622 x_{2} x_{4}
\end{gathered}
$$

\subsubsection{Effect of Each Factor on Ultraweak Luminous Value of Mint}

Fig.(1-3) showed that luminous value of mint increased with illumination and moisture, but decreased with humidity when the other factors were optimum. The maximum luminous value of mint was obtained when 10 lamps were used (mean illumination value was $4229 \mathrm{Lx}$ ), temperature, humidity and moisture were $35^{\circ} \mathrm{C}, 65 \%$ and $90 \mathrm{~mL} / \mathrm{d}$ respectively, and because of the positive correlation between ultraweak luminescence and viability of plants, the viability of mint also reached the maximum.

Luminous value (viability) of mint was higher while illumination increased, that is because light is energy source of photosynthesis, and is the essential growth condition for chloroplast development and chlorophyll synthesis. Light can also regulate the activity of some enzymes in carbon cycle of photosynthesis. At some extent, a linear increase was observed in photosynthetic rate with the increase of illumination. Furthermore, as the main external factor that affects transpiration, illumination facilitates stoma open and reduces stomatal resistance. In addition, illumination increased vapor pressure gradient and transpiration rate by enhancing air temperature and leaf temperature. Consequently, luminous value of mint was elevated with increased illumination, namely viability of mint was increased when illumination increased.

Luminous value (viability) of mint decreased when humidity increased, that was because relative air humidity influences transpiration rate directly. When humidity decreased, vapor pressure decreased, differential pressure inside and outside leaves increased, then transpiration rate increased finally. As the main power of water absorption and transportation, transpiration also facilitates the transmission and distribution of mineral salts and other water-soluble substances in plants. Hence, on the premise of ensuring plants' normal life, slight decrease of humidity promotes photosynthesis rate and viability of plants. In other words, luminous value increased when humidity decreased.

Luminous value (viability) of mint increased with moisture, as a great deal of water was needed in growth of mint. Appropriate irrigation increased the growth rate, enlarged the leaf area and photosynthesis area, and it also improve the activity of roots, absorption of water and mineral substances and photosynthesis rate. Therefore, proper increase of moisture elevated viability and luminescence of mint. 


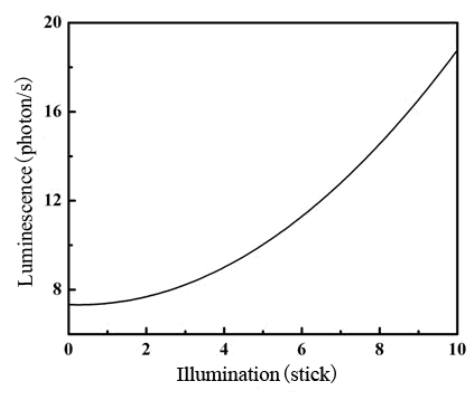

Fig. 1. Effect of illumination on the bio-illumination of mint sample

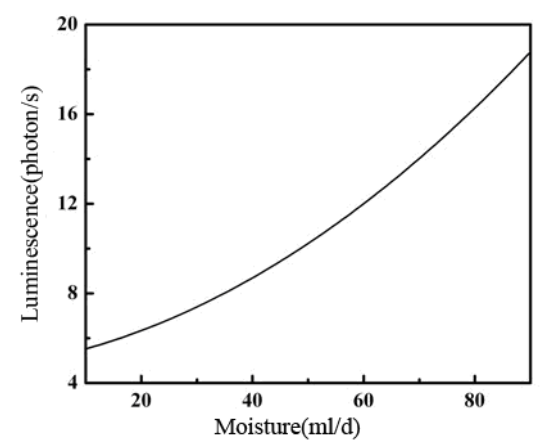

Fig. 3. Effect of moisture on the bio-illumination of mint sample

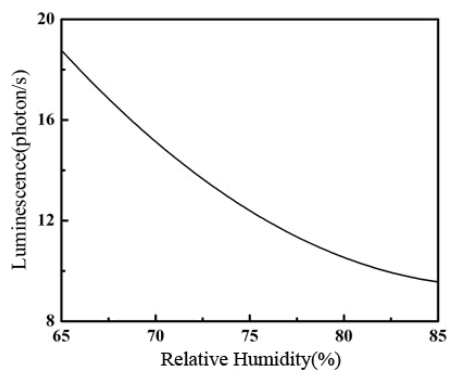

Fig. 2. Effect of relative humidity on the bio-illumination of mint sample

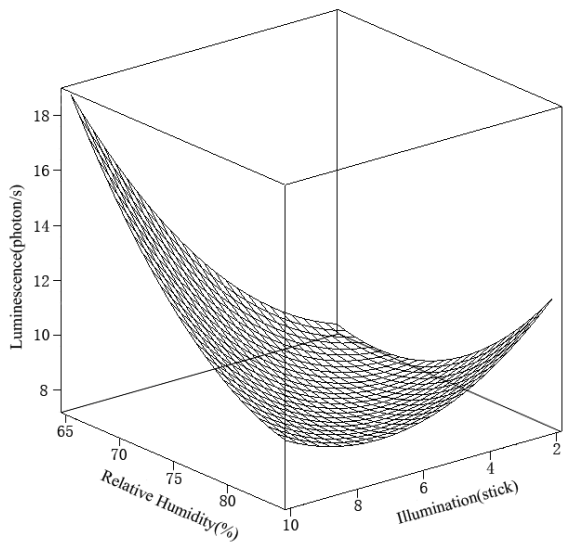

Fig. 4. Effect of relative humidity and illumination on the bio-illumination of mint sample

\subsubsection{Interaction Effect of Three Factors on Ultraweak Luminous Value of Mint}

Fig. 4 showed that the variation trend of luminous value changed with varied illumination. At first, luminous value of mint increased with illumination, with the increase of humidity, luminous value became initially decreased then increased. With higher illumination, similarly, the variation trend of luminescence changed with varied humidity. At first luminescence increased with humidity, with the increase of illumination, luminescence became decreased with humidity. These were explained as different effects that humidity and illumination attached on luminous value of mint, and the interaction between them had significant effect on mint's luminescence (Tab. 1). 


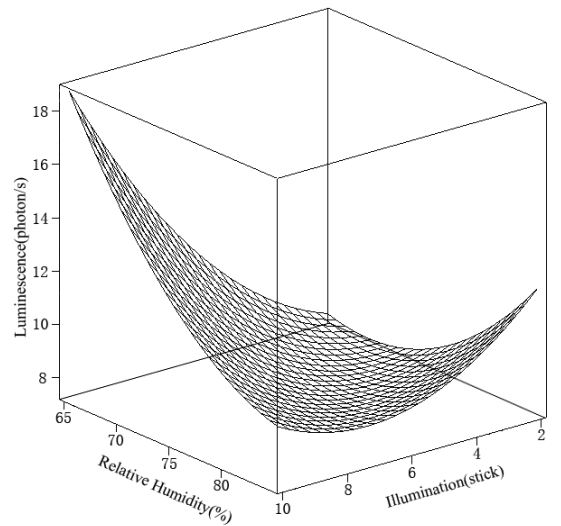

Fig. 5. Effect of moisture and illumination on the bio-illumination of mint sample

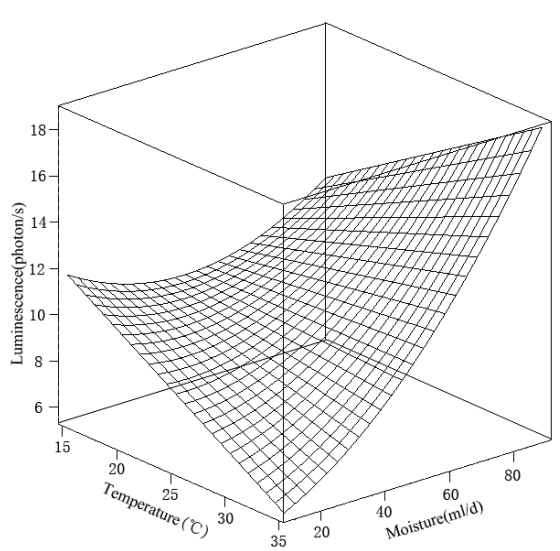

Fig. 6. Effect of temperature and moisture on the bio-illumination of mint sample

Fig. 5 showed that the luminous value changed with moisture, firstly luminous value initially decreased then increased with moisture, with the increase of temperature, luminous value became increased with moisture. Similarly, luminous value of mint decreased with the increase of temperature when moisture was in low level, but with the increase of moisture, luminous value became increased with temperature. It indicated that environmental temperature affected the luminous value, but the impact was performed by the interaction of temperature and moisture, and the interaction had significant effect on luminous value of mint (Tab. 1).

\section{Conclusion}

Single factors and interactions of illumination, temperature, humidity and moisture all have effect on the luminous character of mint leaves. For single factors, moisture and humidity have more significant effect on luminescence of mint than others. The interaction of illumination and humidity has the most significant effect on luminescence. Besides, the mathematical model established in this study effectively reflects the impacts of various environment factors on ultraweak luminous properties of mint.

Acknowledgements. This research was supported by a grant from the Research Fund for the Doctoral Program of Higher Education of China (RFDP) (No. 20090101120081), the major science and technology project of Science Technology Department of Zhejiang Province, China (No. 2008C02006-4), and the project of Education Department of Zhejiang Province, China (No. Y20080327). 


\section{Reference}

1. Ramamoorthy, V., Viswanathan, R., Raguchander, T., Prakasam, V., Samiyappan, R.: Induction of systemic resistance by plant growth promoting rhizobacteria in crop plants against pests and diseases. Crop Protection 20(1), 1-11 (2001)

2. Zhang, X.H., Yang, H.Q.: Ultraweak bioluminescence in plant. Journal of Shandong Agricultural University 34(4), 605-608 (2003)

3. Lie, G.H., Zhao, P.F., Zhang, X.M., Lu, H.B., Jiang, T., Li, W.D.: Laser microbeam technique, biological ultraweak photon emission and biological vitomystery. Journal of Qinghai Mormal University (Natural Science Edition) 1, 78-81 (2003)

4. Zhang, Z.L.: Ultra-weak Chemiluminescence Analytical Technology Principle and Application. Progress in Biochemistry and Biophysics 27(1), 102-104 (2000)

5. Zhang, J.P., Zhang, X.Z., Gong, Z.H.: Application of ultraweak bioluminescence in vegetable research. Chinese Agricultural Science Bulletin 22(1), 220-222 (2006)

6. Cheng, H.P., Wang, J.H., Chi, H.C., Zhu, M.Y.: Study on ultraweak luminescence of pisum sativum seeds at the stage of germination. Journal of Zhejiang University (Science Edition) 28(6), 682-685 (2001)

7. Tan, S.C., Xing, D., Tang, Y.H., Li, D.H.: Spectral studies of ultra-weak biophoton emission from Plant's leabes. Acta Photonica Sinica 29(11), 961-965 (2000)

8. Ma, F., Feng, M., Gao, Y., Feng, X.M., Wang, Z.G., Luo, S.S., Ge, C.L.: Vegetable seed radiosensitivity and kinetic analysis of super-weak bioluminescence. Journal of Nuclear Agricultural Sciences 17(5), 382-387 (2003)

9. Gao, Y., Feng, M., Wang, Z.G., Lu, H.Y., Ma, F., Shi, Z.L., Ge, C.L., Luo, S.S.: A preliminary study of irradiation disturbance on the rapid bacteria assay with ATP bioluminescence technique for agricultural products. Journal of Nuclear Agricultural Sciences 21(1), 48-51 (2007)

10. Chen, W.L., Xing, D., Wang, J., He, Y.: Rapid determination of rice seed vigour by spontaneous chemiluminescence and singlet oxygen generation during early imbibition. Luminescence 18(1), 19-24 (2003)

11. Strand, J.F.: Some agrometeorological aspects of pest and disease management for the $21 \mathrm{st}$ century. Agricultural and Forest Meteorology 103, 73-82 (2000)

12. Yazgan, M.S., Tanik, A.A.: New approach for calculating the relative risk level of pesticides. Environment International 31, 687-692 (2005)

13. Li, Z.H., Li, P.P.: The study of a system for the diagnosis and prevention of pests, diseases and nutritional deficiency of greenhouse tomato. Journal of Agricultural Mechanization Research 2, 187-191 (2003) 